\title{
Escape from an attractor generated by recurrent exit
}

\author{
Lou Zonca $\odot^{1,2}$ and David Holcman ${ }^{1}$ \\ ${ }^{1}$ Group of Data Modeling, Computational Biology and Applied Mathematics, Ecole Normale Supérieure-PSL, 75005 Paris, France \\ ${ }^{2}$ Sorbonne University, Pierre et Marie Curie Campus, 75005 Paris, France
}

(Received 17 September 2020; accepted 5 March 2021; published 14 May 2021)

\begin{abstract}
Kramer's theory of activation over a potential barrier consists in computing the mean exit time from the boundary of a basin of attraction of a randomly perturbed dynamical system. Here we report that for some systems, crossing the boundary is not enough, because stochastic trajectories return inside the basin with a high probability a certain number of times before escaping far away. This situation is due to a shallow potential. We compute the mean and distribution of escape times and show how this result explains the large distribution of interburst durations in neuronal networks.
\end{abstract}

DOI: 10.1103/PhysRevResearch.3.023115

\section{INTRODUCTION}

In Kramers' theory [1-4], the escape time over a potential barrier consists in computing the mean first passage time (MFPT) of a dynamical system perturbed by a small noise to the boundary of a basin of attraction. The MFPT measures the stability and provides great insight into the backward binding rate in chemistry [5,6], loss of lock for phase controllers in communication theory [7], escape of receptors from the postsynaptic density at neuronal synapses, and is also used to evaluate future derivatives in the financial market [8]. The full distribution of exit times can be used to characterize both short and intermediate time asymptotics relevant in polymer physics [9], accelerating chemical reaction simulations [10], or better characterizing the search for a small target in a complex environment [11,12].

In the limit of small noise, a trajectory escapes a basin of attraction with probability 1 [13], but the escape time is exponentially long depending on the topology of the noiseless dynamics $[14,15]$ and its behavior at the boundary. In addition, the distribution of exit points peaks at a distance $O(\sqrt{\sigma})$ from a saddle point, where $\sigma$ is the noise intensity $[2,7,16]$. Interestingly, when a focus attractor is located near the boundary of the basin of attraction, the escape time deviates from an exponential distribution because trajectories oscillate inside the attractor before escape [17-21].

In these previous examples, the escape ends at the first time a trajectory crosses the separatrix that delimits the basin of attraction. Recurrent returns inside a basin of attraction can be quantified by the Green's function of the inner domain used in the additive properties of the MFPT [22]. In their specific case, where the escape time consists in the first crossing of the

Published by the American Physical Society under the terms of the Creative Commons Attribution 4.0 International license. Further distribution of this work must maintain attribution to the author(s) and the published article's title, journal citation, and DOI. boundary of the basin of attraction and a second separatrix, their results show a factor 2 between the escape time and the exit from the basin of attraction. In dimension one, a recurrent return can be quantified using a relaxation time computed from the survival probability when it does not converge to zero in the long times regime [23]. We show here that for some shallow two-dimensional dynamical systems, trajectories can first exit the basin of attraction, then make excursions outside before coming back inside the domain, a behavior that occurs several times before eventually escaping far away. This situation is peculiar and specific to dimensions greater than two and these recurrent entries need to be taken into account in computing the final escape time.

This paper reports such phenomenon. We present formulas for the mean and distribution of escape times and we show that these recurrent reentries inside the basin of attraction can increase the escape time by a factor between 2 and 3. Finally, we apply these results to explain the origin of long interburst durations found in neuronal network models [24].

\section{RECURRENT ESCAPE PATTERNS}

We start with a generic two-dimensional system

$$
\begin{aligned}
\dot{h} & =-\alpha h+x^{2}+\sigma \dot{\omega}, \\
\dot{x} & = \begin{cases}h-\gamma x & \text { for } h \geqslant 0 \\
-\gamma x & \text { for } h \leqslant 0,\end{cases}
\end{aligned}
$$

where $\alpha \in] 0,1], \gamma \in] 0, \alpha[, \dot{\omega}$ is a Gaussian white noise, and $\sigma$ its intensity. The determinist part of this system has two critical points: one attractor $A=(0,0)$ [Fig. 1(a), red star] and one saddle point $S=\left(\gamma^{2} \alpha, \gamma \alpha\right)$ [Fig. 1(a), cyan star], and the separatrix $\Gamma$ delimits the basin of attraction of $A$ [Fig. 1(a), solid black].

The escape of the basin of attraction occurs in two steps. (1) A trajectory starting at $A$ reaches $\Gamma$ for the first time [Fig. 1(a), black trajectory between $A$ and the first exit point Exit no. 1, light green). (2) The trajectory exits and crosses $\Gamma$ several times, which we count by using a round-trip (RT) number [Fig. 1(a), light-green and cyan loops] before eventually 

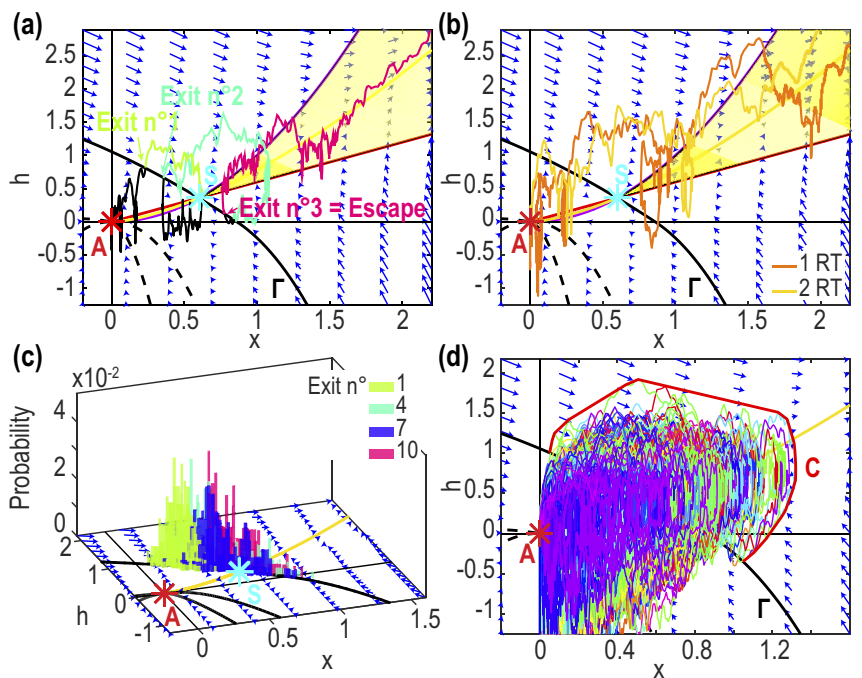

FIG. 1. Recurrent escape patterns. (a) Escaping trajectories reach the separatrix $\Gamma$ for the first time (step 1, black) and recross it several times going back and forth inside and outside of the basin of attraction (step 2 green, cyan) before eventually escaping far away (pink). (b) Stochastic trajectories with one (yellow) and two (orange) round-trips (RTs) before escape. (c) Distributions of exit points on $\Gamma$ (500 runs) for successive RTs. (d) Outer boundary layer $C$ computed as the convex hull of all trajectories reentering the basin of attraction (red).

escaping far away [Fig. 1(a), pink]. To characterize the final escape times and the distribution of crossing points on $\Gamma$, we ran stochastic simulations of system (1) (five hundred runs) [Fig. 1(b), trajectories exhibit one (yellow) and two (orange) RTs before escape]. To further characterize the recurrent crossing points, we plotted their distributions [Fig. 1(c)] and found that they were peaked near the saddle point. These recurrent excursions are not due to a focus, since the saddle point $S$ has only real eigenvalues $\lambda_{ \pm}=-\frac{1}{2}\left[-(\alpha+\gamma) \pm \sqrt{(\alpha+\gamma)^{2}+4 \alpha \gamma}\right]$, $\lambda_{+} \approx 0.314, \lambda_{-} \approx-1.914$ (with $\alpha=1$ and $\gamma=0.6$ ). A possible explanation for this phenomenon is the very shallow field tangent to the separatrix: only near the unstable manifold [Fig. 1(a), yellow curve] trajectories can depart to infinity when they are located inside the ensemble of points where the two drift components are positive $\dot{x}>0$ and $\dot{h}>0$, thus $C_{\infty}=\left\{h>\gamma x\right.$ and $\left.h<\frac{x^{2}}{\alpha}\right\}$ [Figs. 1(a) and 1(b), yellow area, situated between the $x$ nullcline (red) and the $h$ nullcline (purple)]. Before reaching $C_{\infty}$, the noise pushes the trajectories back and forth into the basin of attraction.

To conclude this part we shall summarize the escape dynamics:

(1) The distribution of exit points peaks at a distance $O(\sqrt{\sigma})$ from the saddle point (generically satisfied [16]).

(2) The shallow field near the separatrix allows the trajectories to reenter with high probability.

(3) The peaks of the successive exit point distributions drift towards the saddle point $S$ [Fig. 1(c)].

(4) When the trajectories enter the escape cone $C_{\infty}$ [yellow surface in Figs. 1(a) and 1(b)] where the field increases, they eventually escape to infinity.
Finally, this escape pattern could not occur in dimension one since conditions (1) and (3) cannot be satisfied.

\section{CHARACTERIZING THE ESCAPE TIME}

We compute here the total escape time. For that goal, we decomposed it into the time to reach the separatrix $\Gamma$ for the first time plus the time spent to go back and forth around $\Gamma$ before the final escape. Using Baye's law and conditioning on the RT numbers, the mean escape time can be written as

$$
\left\langle\tau_{\mathrm{esc}}\right\rangle=\sum_{k=0}^{\infty}\langle\tau \mid k\rangle P_{R T}(k),
$$

where $\langle\tau \mid k\rangle$ [respectively, $P_{R T}(k)$ ] is the mean time (respectively, probability) to return $k$ times inside the basin of attraction. To estimate the escape probability $\tilde{p}$ for a trajectory that had crossed $\Gamma$ to escape to infinity, we ran $N=500$ trajectories starting from $A$ and lasting $T=300 \mathrm{~s}$. We first counted the proportion of trajectories reentering the basin of attraction at least once and obtained $88 \%$. We then reiterated this process and counted the proportion of trajectories reentering the basin of attraction one more time after each RT. We found that this proportion was stable equal to $88 \%$, leading to $\tilde{p}=0.12$. We applied this process for values of the noise intensity $\sigma \in[0.21,1.05]$ and found that $\tilde{p}$ did not depend on $\sigma$. After $T=300 \mathrm{~s}$ all trajectories had escaped to infinity (for all the values of $\sigma$ ), thus choosing a higher value for $T$ would not change the value of $\tilde{p}$. This escape phenomenon could be interpreted as follows: a trajectory has escaped when it reaches a distance far away from the separatrix and to better characterize such a distance outside the basin of attraction, we generated empirical trajectories that will return (have not yet escaped) and estimated their convex hull $C$ [Fig. 1(d), red, 500 runs]. Formally, this is equivalent to looking at trajectories starting at $A$ conditioned to a return to the basin of attraction, thus defining a sort of Brownian bridge. This procedure leads to a bounded domain: any point inside $C$ has a high probability of reentering the basin of attraction while points further away will escape to infinity.

Due to the strong Markovian properties, each RT can be considered independent of the previous ones, thus the probability to escape after exactly $k-\mathrm{RT}$ is given by

$$
P_{R T}(k)=\tilde{p}(1-\tilde{p})^{k-1},
$$

and thus the mean escape time is

$$
\begin{aligned}
\left\langle\tau_{\text {esc }}\right\rangle & =\left\langle\tau_{0}\right\rangle+\left(\left\langle\tau_{\text {ext }}\right\rangle+\left\langle\tau_{\text {int }}\right\rangle\right) \tilde{p} \sum_{k=1}^{\infty} k(1-\tilde{p})^{k-1} \\
& =\left\langle\tau_{0}\right\rangle+\frac{\left\langle\tau_{\text {ext }}\right\rangle+\left\langle\tau_{\text {int }}\right\rangle}{\tilde{p}},
\end{aligned}
$$

where $\left\langle\tau_{0}\right\rangle$ is the mean time to reach the separatrix for the first time and $\left\langle\tau_{\text {ext }}\right\rangle$ (respectively, $\left.\left\langle\tau_{\text {int }}\right\rangle\right)$ is the time spent on the outside (respectively, inside) of the basin of attraction of $A$ for each RT [Fig. 2(a)]. When the escape probability $\tilde{p}$ tends to zero, the escape time tends to infinity, corresponding to trajectories that would be trapped in $C$. In our case, the mean escape time is $\left\langle\tau_{\text {esc }}\right\rangle \approx\left\langle\tau_{0}\right\rangle+8.33\left(\left\langle\tau_{\text {ext }}\right\rangle+\left\langle\tau_{\text {int }}\right\rangle\right)$. With 

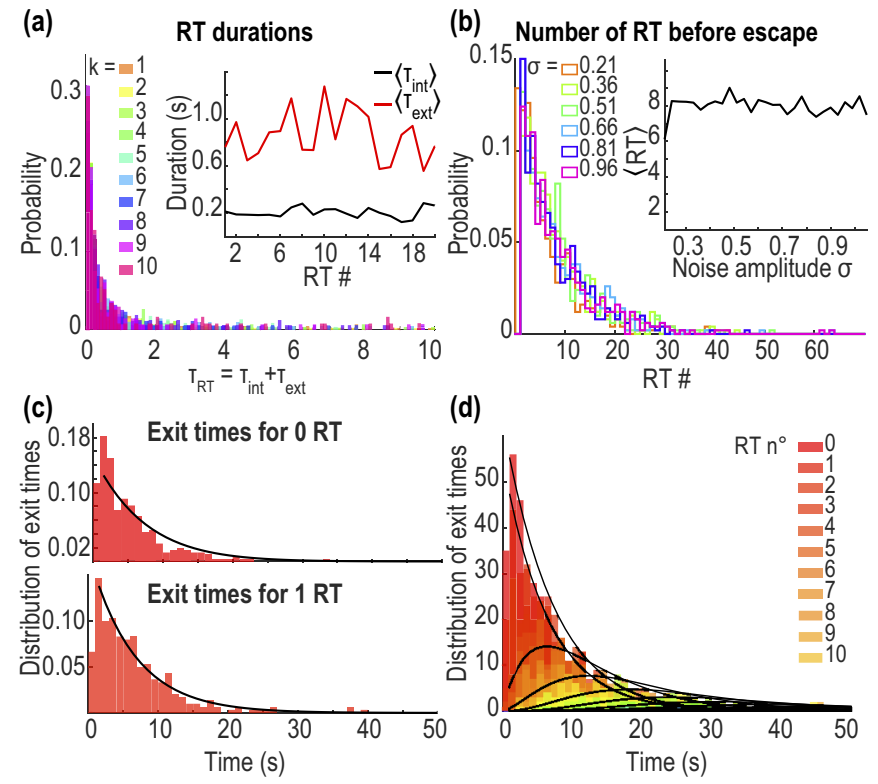

FIG. 2. Distribution of RT and escape times. (a) Distributions of a RT duration $\tau_{R T, k}=\tau_{\text {ext }, k}+\tau_{\text {int }, k}$ for $k \in[1,10]$. Inset: mean time spent outside (respectively, inside) the basin of attraction $\left\langle\tau_{\mathrm{ext}, k}\right\rangle$ (respectively, $\left\langle\tau_{\text {int }, k}\right\rangle$ ) vs the RT number $k$. (b) Distributions of the RT number around the separatrix before a trajectory eventually escapes for various values of $\sigma$ (with $\gamma=0.6$ and $\alpha=1$ ). Five hundred runs for each value of $\sigma$. Inset: mean RT number with respect to the noise intensity $\sigma$. (c) Distributions $f_{0}$ (upper), respectively, $f_{1}$ (lower), of escape times for trajectories with zero and one RT. The fit uses Eq. (8). (d) Distribution of exit times with the contribution of each RT number compared to the analytical distribution [Eq. (7)].

the present parameters $\left\langle\tau_{0}\right\rangle \approx 5.1 \mathrm{~s}$ and $\left\langle\tau_{\text {ext }}\right\rangle+\left\langle\tau_{\text {int }}\right\rangle \approx 1 \mathrm{~s}$ showing that the escape time is increased by a factor 2.6. Interestingly, the noise intensity does not influence the number of RTs before escape [Fig. 2(b)]. For the parameter value $\gamma=0.6$, we found that a trajectory performs eight RTs on average [Fig. 2(b), inset]. These results indicate that the noise intensity does not directly influence the probability to escape to infinity. We now determine the distribution of escape times

$$
P\left(\tau_{\mathrm{esc}}<t\right)=\sum_{k=0}^{\infty} P\left(\tau^{k}<t \mid k\right) P_{R T}(k),
$$

where $P\left(\tau^{k}<t \mid k\right)$ is the conditional probability distribution to escape after $k$ RTs. Because RTs are independent and identically distributed, this probability is the $k$ th convolution of the distribution of times of a single RT $f_{1}(t)$ with the distribution of escape times without RT $f_{0}(t)$

$$
P\left(\tau^{k}<t \mid k\right)=f_{0}(t) * f_{1}(t)^{* k}
$$

where $f(t)^{* k}=f(t) * f(t) * \cdots * f(t), k$ times. Thus the probability density function (pdf) of exit times is given by

$$
f(t)=\sum_{k=0}^{\infty} f_{0}(t) * f_{1}(t)^{* k} \tilde{p}(1-\tilde{p})^{k-1} .
$$

To compare this formula to the results of our numerical simulations, we approximate the distributions $f_{0}$ and $f_{1}$ by

$$
f_{i}(t)=c_{i}\left[1+\operatorname{erf}\left(\frac{t-a_{i}}{b_{i}}\right)\right] e^{-\lambda_{i} t}, \text { for } i=0,1,
$$

where $\operatorname{erf}(x)=\frac{2}{\sqrt{\pi}} \int_{0}^{x} e^{-u^{2}} d u$ is the error function. We fitted the distributions obtained from the numerical simulations of trajectories that escaped without doing any RT $\left[f_{0}\right.$ Fig. 2(c), upper] and after one single RT [ $f_{1}$ Fig. 2(c), lower] with the condition that $\lambda_{1} \geqslant \lambda_{0}$. We obtained $c_{0}=1.09, c_{1}=1.63$, $\lambda_{0}=0.06, \lambda_{1}=0.13, a_{0}=-38.28, a_{1}=-138.64, b_{0}=$ $-36.72, b_{1}=-121.69$. We then computed each term of the sum (7) and we could compare it to the corresponding parts of the distribution of escape times obtained from stochastic simulations [Fig. 2(d)].

\section{INTERBURST DURATIONS IN A FIRING EXCITATORY NEURONAL NETWORK}

Burst and interburst are fundamental network events occurring during dominant imbalance dominated by excitatory neuronal activity. Network burst generation could rely on specific spiking frequencies in connected neurons [25] despite a high variability in interspike intervals [26]. Neuronal population bursts separated by long interbursts have been modeled using a two-state synaptic depression [27], or by using the refractory period induced by afterhyperpolarization (AHP), a mechanism leading to a long voltage hyperpolarization transient and generated by various potassium channels [28]. Here we show that the recurrent escape mechanism described above can be used as one explanation of the origin of long interburst intervals without the need of any other mechanism. However, we note that this mechanism does not have to be exclusive and that long interburst intervals could also be explained in some cases by a combination of mechanisms such as the recurrent escape pattern presented here and AHP. Indeed, we start from the depression-facilitation short-term synaptic plasticity mean-field model of network neuronal bursting [29-31], which consists of three equations (9) for the mean voltage $h$, the depression $y$, and the facilitation $x$. The depression mechanism describes the depletion of the vesicular pool necessary for neurotransmission following successive action potentials, while the facilitation mechanism corresponds to a transient increase of the release probability mediated by a local calcium accumulation at synapses.

$$
\begin{aligned}
\tau \dot{h} & =-h+J x y h^{+}+\sqrt{\tau} \sigma \dot{\omega}, \\
\dot{x} & =\frac{X-x}{t_{f}}+K(1-x) h^{+}, \\
\dot{y} & =\frac{1-y}{t_{r}}-L x y h^{+},
\end{aligned}
$$

where $h^{+}=\max (h, 0)$ is a linear threshold function of the synaptic current that gives the average population firing rate $[29,31,32]$. The mean number of connections (synapses) per neuron is accounted for by the parameter $J$ and the term $J x y$ represents the combined effect of the short-term synaptic plasticity (facilitation and depression mechanisms) on the network activity. The parameters $K$ and $L$ describe how the firing rate 
TABLE I. Model (9) parameters,

\begin{tabular}{llc}
\hline \hline & \multicolumn{1}{c}{ Parameters } & Values \\
\hline$\tau$ & Time constant for $h$ & $0.05 \mathrm{~s} \mathrm{[28]}$ \\
$J$ & Synaptic connectivity & $4.21[28]$ \\
$K$ & Facilitation rate & $0.037 \mathrm{~Hz}[28]$ \\
$X$ & Facilitation resting value & $0.08825[28]$ \\
$L$ & Depression rate & $0.028 \mathrm{~Hz}[28]$ \\
$\tau_{r}$ & Depression time rate & $2.9 \mathrm{~s} \mathrm{[28]}$ \\
$\tau_{f}$ & Facilitation time rate & $0.9 \mathrm{~s} \mathrm{[28]}$ \\
$T$ & Depolarization parameter & 0 \\
\hline \hline
\end{tabular}

is transformed into molecular events that are changing the duration (depression) and probability (facilitation) of vesicular release. The timescales $t_{f}$ and $t_{r}$ define the recovery of an averaged synapse from the network activity. Finally, $\dot{\omega}$ is an additive Gaussian noise and $\sigma$ its intensity; this additive noise term represents the fluctuations of the mean voltage generated by the average of independent vesicular release events and/or closings and openings of voltage gated channels.

This system has three critical points: one attractor and two saddles. Interestingly, near the attractor $A=(0, X, 1)$, the dynamic is anisotropic $\left(\left|\lambda_{1}\right|=12.6 \gg\left|\lambda_{2}\right|=1.11 \gg\left|\lambda_{3}\right|=\right.$ 0.34 , with the parameters from Table I) and thus we project the system on the two-dimensional plan $y=C t e$

$$
\dot{y}=0=\frac{1-y}{\tau_{r}}-L x y h^{+}=0 \Longleftrightarrow y=\frac{1}{1+\tau_{r} L x h^{+}}
$$

leading to the simplified system

$$
\begin{aligned}
& \dot{h}=\frac{h\left(J x-1-\tau_{r} L x h^{+}\right)}{\tau\left(1+\tau_{r} L x h^{+}\right)}+\sqrt{\tau} \sigma \dot{\omega}, \\
& \dot{x}=\frac{X-x}{\tau_{f}}+K(1-x) h^{+} .
\end{aligned}
$$

The deterministic component of this system has three critical points: two attractors and one saddle point.

\section{A. Attractor $A_{0}$}

A first equilibrium point is given by $h=0$ and $x=X$. The Jacobian at this point is

$$
J_{A}=\left(\begin{array}{cc}
\frac{-1+J X}{\tau} & 0 \\
K(1-X) & -\frac{1}{\tau_{f}}
\end{array}\right) .
$$

With our parameters (Table I) the eigenvalues $\lambda_{1}=\frac{J X-1}{\tau} \approx$ -12.6 and $\lambda_{2}=-\frac{1}{\tau_{f}} \approx-1.11$ are both negative, confirming $A$ is an attractor.

\section{B. Saddle point $S$}

The second critical point is $S_{1}\left(h_{1} \approx 8.07 ; x_{1} \approx 0.28\right)$. Its eigenvalues are $\lambda_{1} \approx-5.73$ and $\lambda_{2} \approx 1.43$. It is a saddle point.

\section{Attractor $A_{2}$}

The third critical point is $A_{2}\left(h_{2} \approx 28.8 ; x_{2} \approx 0.53\right)$. Its eigenvalues are $\lambda_{1} \approx-11.9$ and $\lambda_{2} \approx-1.33$. It is another
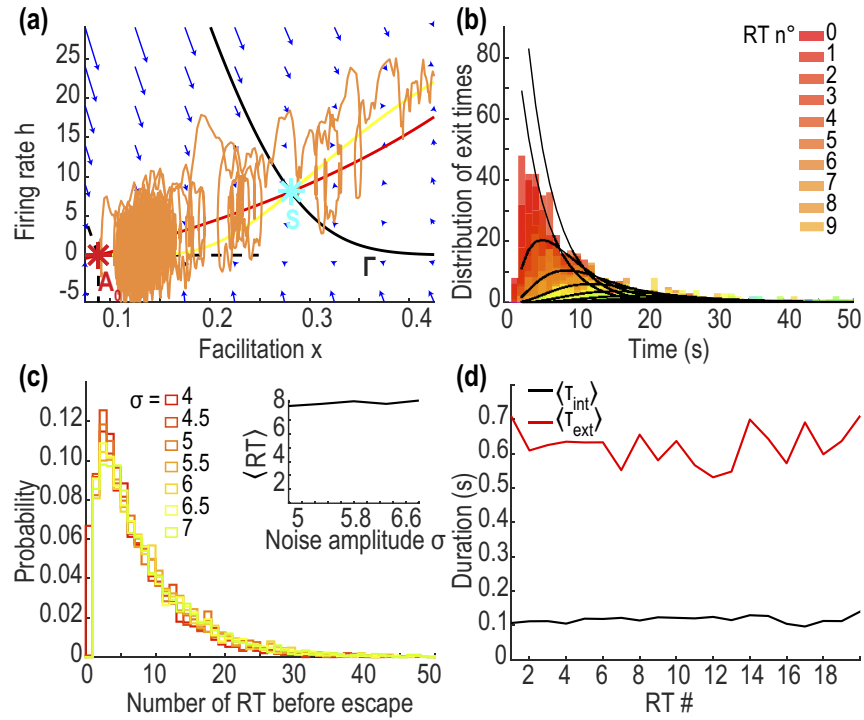

FIG. 3. Application to the dynamical system (11). (a) Twodimensional phase space restricted to $\{x \leqslant 0.5$ and $h \leqslant 30\}$. The basin of attraction of $A_{0}$ (red star) is delimited by the stable manifold of $S$ (solid black curve $\Gamma$ ) with an exiting trajectory doing one RT (orange) around the separatrix before escape.(b) Distribution of exit times with the contribution of the trajectories per RT number before escape with the analytical fit [Eqs. (7), (13), and (14)]. (c) Distribution of the RT number for $\sigma \in[4,7]$ and mean RT number with respect to noise (inset). (d) Values of $\left\langle\tau_{\text {ext }}\right\rangle$ (red) and $\left\langle\tau_{\text {int }}\right\rangle$ (black) with respect to the RT number.

attractor. The two attractors are separated by the onedimensional stable manifold of the saddle point $S_{1}$ [Fig. 3(a), solid black curve].

The phase space of system (11), restricted to the region $\{x \leqslant 0.5$ and $h \leqslant 30\}$ has the same topological properties as system (1): one attractor and one saddle point; the separatrix delimiting the basin of attraction is the stable manifold of $S_{1}$ [Fig. 3(a)]. The escaping trajectory exits and reenters the basin of attraction several times before eventually escaping [Fig. 3(a), orange].

Thus, we can now understand that the interburst intervals correspond to the exit times of trajectories from the basin of attraction. Using formula (7) to fit the distribution of exit times, we obtain that $\tilde{p} \approx 0.13$ [Fig. 3(b)] and

$$
f_{0}(t)=0.23 \exp (-0.25 t)\left[1+\operatorname{erf}\left(\frac{t-2.45}{0.43}\right)\right]
$$

and

$$
f_{1}(t)=0.19 \exp (-0.25 t)\left[1+\operatorname{erf}\left(\frac{t+15.97}{0.58}\right)\right] .
$$

Finally, similar to the generic system (1), the RT number before escape does not depend on the noise intensity [Fig. 3(c)]. Trajectories are making on average eight RTs before escape (inset). To determine the mean escape time, we use formula (4) and obtain $\left\langle\tau_{\text {esc }}\right\rangle \approx\left\langle\tau_{0}\right\rangle+7.7\left(\left\langle\tau_{\text {ext }}\right\rangle+\left\langle\tau_{\text {int }}\right\rangle\right)$ where $\left\langle\tau_{0}\right\rangle \approx 4.35 \mathrm{~s}$ and $\left\langle\tau_{\text {ext }}\right\rangle+\left\langle\tau_{\text {int }}\right\rangle \approx 0.7 \mathrm{~s} \mathrm{[Fig.} \mathrm{3(d)]} \mathrm{thus} \mathrm{leading}$ to a factor 2.2 in the escape time. At this stage we conclude that long interburst durations, generated by excitatory neu- 
ronal networks [33], can be explained by the recurrent escape mechanism introduced here.

\section{CONCLUDING REMARKS}

We presented an escape mechanism for which reaching the boundary of the deterministic basin of attraction induced by noise is not sufficient to escape. After crossing the separatrix, the noise tends to bring trajectories back inside the basin of attraction until they reach a region (escape conelike domain $C_{\infty}$ ), narrow near $S$ and that widens with the distance. The size of the characteristic distance from $S$ (boundary layer) after which trajectories escape is $\sqrt{\frac{\lambda_{+}}{\sigma}}$ [34]. We derived formulas for the mean escape time and the distribution of escape times taking into account the excursions inside and outside of the basin of attraction before the final escape.

\section{NUMERICAL METHODS}

All simulations were run in Matlab using the Runge-Kutta 4 scheme with a time step $\delta_{t}=0.01 \mathrm{~s}$ (we also tried $\delta_{t}=$ $0.001 \mathrm{~s}$ and obtained the same results thus ensuring stability). The same results were also obtained using the Euler method.

\section{ACKNOWLEDGMENTS}

L.Z. received funding from French Research Ministry (ED386 Ecole doctorale de Sciences Mathématiques Paris centre) and Fondation pour la Recherche Médicale (FRM - FDT202012010690). This project has received funding from the European Research Council (ERC) under the European Union's Horizon 2020 research and innovation program (Grant Agreement No. 882673).
[1] H. A. Kramers, Brownian motion in a field of force and the diffusion model of chemical reactions, Physica 7, 284 (1940).

[2] Z. Schuss, Theory and Applications of Stochastic Differential Equations (Wiley, New York, 1980).

[3] Z. Schuss, Theory and Applications of Stochastic Processes: An Analytical Approach (Springer New York, 2010).

[4] C. Gardiner, Handbook of Stochastic Methods for Physics, Chemistry, and the Natural Sciences, Proceedings in Life Sciences (Springer-Verlag, Berlin, 1985).

[5] M. I. Dykman, E. Mori, J. Ross, and P. Hunt, Large fluctuations and optimal paths in chemical kinetics, J. Chem. Phys. 100, 5735 (1994).

[6] A. Nitzan, Chemical Dynamics in Condensed Phases: Relaxation, Transfer and Reactions in Condensed Molecular Systems (Oxford University Press, New York, 2006).

[7] Z. Schuss, Nonlinear Filtering and Optimal Phase Tracking (Springer Science \& Business Media, Berlin, 2011), Vol. 180.

[8] J.-P. Fouque, G. Papanicolaou, and K. R. Sircar, Derivatives in Financial Markets with Stochastic Volatility (Cambridge University Press, Cambridge, UK, 2000).

[9] A. T. Hawk, S. S. M. Konda, and D. E. Makarov, Computation of transit times using the milestoning method with applications to polymer translocation, J. Chem. Phys. 139, 064101 (2013) [JCP: BioChemical Physics 7, 08B611_1 (2013)].

[10] C. Dellago, P. G. Bolhuis, and D. Chandler, Efficient transition path sampling: Application to Lennard-Jones cluster rearrangements, J. Chem. Phys. 108, 9236 (1998).

[11] A. Godec and R. Metzler, Universal Proximity Effect in Target Search Kinetics in the Few-Encounter Limit, Phys. Rev. X 6, 041037 (2016).

[12] D. S. Grebenkov, R. Metzler, and G. Oshanin, Full distribution of first exit times in the narrow escape problem, New J. Phys. 21, 122001 (2019).

[13] B. J. Matkowsky and Z. Schuss, The exit problem for randomly perturbed dynamical systems, SIAM J. Appl. Math. 33, 365 (1977).

[14] M. I. Freidlin and A. D. Wentzell, Random perturbations, in Random Perturbations of Dynamical Systems (Springer, New York, 1998), pp. 15-43.
[15] V. N. Smelyanskiy, M. I. Dykman, and B. Golding, Time Oscillations of Escape Rates in Periodically Driven Systems, Phys. Rev. Lett. 82, 3193 (1999).

[16] B. Bobrovsky and Z. Schuss, A singular perturbation method for the computation of the mean first passage time in a nonlinear filter, SIAM J. Appl. Math. 42, 174 (1982).

[17] T. Verechtchaguina, I. M. Sokolov, and L. Schimansky-Geier, First passage time densities in resonate-and-fire models, Phys. Rev. E 73, 031108 (2006).

[18] T. Verechtchaguina, I. Sokolov, and L. Schimansky-Geier, First passage time densities in non-Markovian models with subthreshold oscillations, Europhys. Lett.) 73, 691 (2006).

[19] T. Verechtchaguina, I. Sokolov, and L. Schimansky-Geier, Interspike interval densities of resonate and fire neurons, Biosystems 89, 63 (2007).

[20] H. C. Tuckwell, J. Jost, and B. S. Gutkin, Inhibition and modulation of rhythmic neuronal spiking by noise, Phys. Rev. E 80, 031907 (2009).

[21] K. Dao Duc, Z. Schuss, and D. Holcman, Oscillatory survival probability: Analytical and numerical study of a non-Poissonian exit time, Multiscale Model. Simul. 14, 772 (2016).

[22] B. Matkowsky, Z. Schuss, and C. Tier, Uniform expansion of the transition rate in Kramers' problem, J. Stat. Phys. 35, 443 (1984).

[23] N. V. Agudov and A. N. Malakhov, Decay of unstable equilibrium and nonequilibrium states with inverse probability current taken into account, Phys. Rev. E 60, 6333 (1999).

[24] S. Coombes and P. C. Bressloff, Bursting: The Genesis of Rhythm in the Nervous System (World Scientific, Singapore, 2005).

[25] B. Ermentrout, M. Pascal, and B. Gutkin, The effects of spike frequency adaptation and negative feedback on the synchronization of neural oscillators, Neural Comput. 13, 1285 (2001).

[26] B. S. Gutkin and G. B. Ermentrout, Dynamics of membrane excitability determine interspike interval variability: A link between spike generation mechanisms and cortical spike train statistics, Neural Comput. 10, 1047 (1998).

[27] C. Guerrier, J. A. Hayes, G. Fortin, and D. Holcman, Robust network oscillations during mammalian respiratory rhythm 
generation driven by synaptic dynamics, Proc. Natl. Acad. Sci. USA 112, 9728 (2015).

[28] L. Zonca and D. Holcman, Modeling bursting in neuronal networks using facilitation-depression and afterhyperpolarization, Commun. Nonlinear Sci. Numer. Simul. 94, 105555 (2020).

[29] M. V. Tsodyks and H. Markram, The neural code between neocortical pyramidal neurons depends on neurotransmitter release probability, Proc. Natl. Acad. Sci. USA 94, 719 (1997).

[30] K. Dao Duc, C.-Y. Lee, P. Parutto, D. Cohen, M. Segal, N. Rouach, and D. Holcman, Bursting reverberation as a multiscale neuronal network process driven by synaptic depression-facilitation, PLoS One 10, e0124694 (2015).

[31] D. Holcman and M. Tsodyks, The emergence of up and down states in cortical networks, PLoS Comput. Biol. 2, 174 (2006).

[32] O. Barak and M. Tsodyks, Persistent activity in neural networks with dynamic synapses, PLoS Comput. Biol. 3, e35 (2007).

[33] O. Chever, E. Dossi, U. Pannasch, M. Derangeon, and N. Rouach, Astroglial networks promote neuronal coordination, Sci. Signaling 9, ra6 (2016).

[34] Z. Schuss, Diffusion and Stochastic Processes. An Analytical Approach (Springer-Verlag, New York, 2009). 